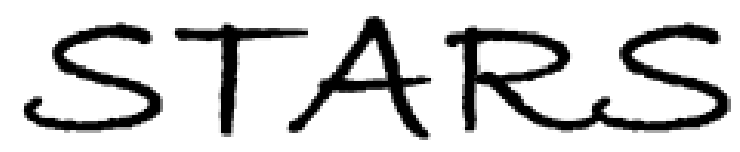

University of Central Florida

STARS

$1-1-2014$

\title{
Studying the effect of zeolite inclusion in aluminum alloy on measurement of its surface hardness using laser-induced breakdown spectroscopy technique
}

Osama Mostafa Khalil

Ilya Mingareev

University of Central Florida

Tobias Bonhoff

University of Central Florida

Ashraf F. El-Sherif

University of Central Florida

Madtistrail|Biquarks@in: https://stars.library.ucf.edu/facultybib2010

University of fentral-Floridalda Libraries http://library.ucf.edu

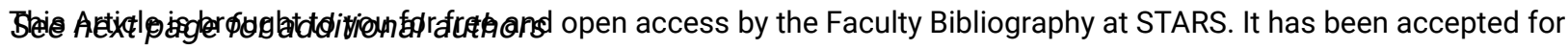
inclusion in Faculty Bibliography 2010 s by an authorized administrator of STARS. For more information, please contact STARS@ucf.edu.

\section{Recommended Citation}

Khalil, Osama Mostafa; Mingareev, llya; Bonhoff, Tobias; El-Sherif, Ashraf F.; Richardson, Martin C.; and Harith, Mohamed Abdel, "Studying the effect of zeolite inclusion in aluminum alloy on measurement of its surface hardness using laser-induced breakdown spectroscopy technique" (2014). Faculty Bibliography 2010s. 5566.

https://stars.library.ucf.edu/facultybib2010/5566

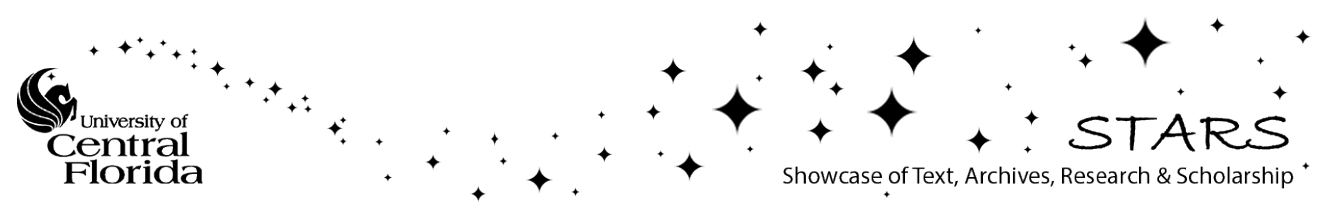




\section{Authors}

Osama Mostafa Khalil, llya Mingareev, Tobias Bonhoff, Ashraf F. El-Sherif, Martin C. Richardson, and Mohamed Abdel Harith 


\section{Optical Engineering}

Studying the effect of zeolite inclusion in aluminum alloy on measurement of its surface hardness using laserinduced breakdown spectroscopy technique

Osama Mostafa Khalil

Ilya Mingareev

Tobias Bonhoff

Ashraf F. El-Sherif

Martin C. Richardson

Mohamed Abdel Harith 


\title{
Studying the effect of zeolite inclusion in aluminum alloy on measurement of its surface hardness using laser-induced breakdown spectroscopy technique
}

\author{
Osama Mostafa Khalil, ${ }^{a}{ }^{\text {I }}$ llya Mingareev, ${ }^{b}$ Tobias Bonhoff, ${ }^{b}$ Ashraf F. El-Sherif, ${ }^{b}$ Martin C. Richardson, ${ }^{b}$ and \\ Mohamed Abdel Harith ${ }^{\mathrm{a}}$ \\ ${ }^{a}$ Cairo University, National Institute of Laser Enhanced Science, P.O. Box 12316, Giza, Egypt \\ bUniversity of Central Florida, The College of Optics and Photonics, P.O. Box 162700, Orlando, Florida
}

\begin{abstract}
Laser-induced breakdown spectroscopy (LIBS) has been used to study the surface hardness of special aluminum alloys containing zeolite. The aluminum alloy has acquired pronounced changes in its metallurgical properties due to the zeolite inclusion. The surface hardness of the samples under investigation is determined by measuring the spectral intensity ratios of the ionic to atomic spectral lines in the LIBS spectra of samples having different surface hardness values that have been conventionally measured before for comparison. The presence of aluminum silicate mineral in the studied alloys enabled material volume to expand under compression. This feature gave new results in the measurement of hardness via LIBS. It has been proven that the trend of the alloy density change complies with the increase of ionic to atomic spectral line intensity ratio. (C) 2014 Society of Photo-Optical Instrumentation Engineers (SPIE) [DOI: 10.1117/1.OE.53.1.014106]
\end{abstract}

Keywords: aluminum alloys; zeolite; laser-induced breakdown spectroscopy; surface hardness.

Paper 131321P received Aug. 28, 2013; revised manuscript received Dec. 7, 2013; accepted for publication Dec. 10, 2013; published online Jan. 24, 2014

\section{Introduction}

The behavior of aluminum alloys under compressive loading does not receive the attention given to tensile properties, perhaps because the strength of structural members is so often limited by buckling. ${ }^{\square}$ For most engineering purposes, it is common to use the same design stress data for a compressive load as for the tensile one. In the testing machine, an aluminum alloy will show an apparently higher strength in compression than in tension, but this can in part be attributed to the cross-sectional areas' change of the specimens, increasing in one case and decreasing in the other, while the stress is based on the original area. It is worthwhile to mention that the mechanical, physical, and casting properties of cast aluminum alloys make them attractive for use in cost-effective, lightweight engineering components. However, to successfully use such alloys in components intended for long-life applications, it is necessary to understand their fatigue resistance. ${ }^{\mathbb{B}}$ From the metallurgical point of view, the effect of hardening by compression changes the sample total volume and consequently its density.

Most materials get compacted or fall apart under pressure, but scientists working in an international collaboration between the U.S. Department of Energy's Brookhaven National Laboratory and the School of Chemical Sciences at England's University of Birmingham have discovered that some expand. These unusual materials may have applications as "molecular sponges" for soaking up chemical pollutants or even radioactive waste. ${ }^{\text {T }}$ This material contains aluminum, silicon, and oxygen with a three-dimensional structure containing regularly spaced pores within the molecular framework, and it is named "zeolite." It is also

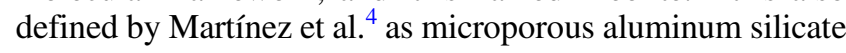

${ }^{*}$ Address all correspondence to: Osama Mostafa Khalil, E-mail: Osama khalil@oi.edu.eg minerals. These nano- or micro-pores make zeolites very useful for sucking up small molecules, ions, or gases, just like a sponge sucking up water. The pores are normally filled with positively charged ions, such as calcium or sodium, and water molecules. So zeolites are said to be hydrated. Many zeolites are currently used as water softeners and in detergents. As for laser interaction with aluminum containing zeolite, it is well known that the change in density due to zeolite inclusion in aluminum alloys affects its heat diffusivity and in turns its vulnerability to laser beam.

Shortly after the invention of the laser in 1960s, researchers found that the beam from a laser could melt and vaporize small amounts of material, especially when the beam is focused onto the surface of an absorbing material. In the early 1970s, laser processing advanced further with the development of multikilowatt $\mathrm{CO}_{2}$ lasers, which led to deep penetration mode of operation. Almost at the same

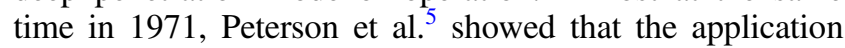
of laser processing increased steadily and described the current status of material processing with lasers before 1971.9 In 1978, Ready nomena behind laser material processing and discussed a broad overview of the field of operation and the effect of different types of lasers and mode of operation on the parameters of laser material processing. In 1999, Xu et al. ${ }^{\mathbb{B}}$ carried out laser transformation hardening experiments on three model steel alloys of nominal composition using continuous wave laser with a near-Gaussian beam. It has been shown that it is possible to isolate the effects of alloy chemistry and beam mode on the kinetics of the hardening process

Laser-induced breakdown spectroscopy (LIBS) is a wellknown spectrochemical elemental analysis technique. It has been found that there is a remarkable correlation between the 
ionic to atomic spectral lines' emission ratio and the surface hardness of solid targets. The relation between the target surface hardness and the ionic to atomic spectral lines' intensity ratio of Zirconium in specially prepared steel alloys treated thermally to have different surface hardnesses has been estimated via LIBS The simplicity and easiness of the LIBS technique in addition to its other advantages compared with other conventional elemental analysis techniques made it exploitable in the field of surface hardness measurements. Moreover, it has been shown that with compact portable LIBS systems, the technique is promising for in situ measurements of solid samples surface hardness of any geometrical shape.

The aim of the present work is to study the effect of inclusion of zeolite in aluminum alloy samples' on surface hardness measured by LIBS. The effect of volume expansion under compression on the relation between the surface hardness and the ionic to atomic spectral lines emission intensity ratio will be demonstrated.

\section{Materials and Methods}

\subsection{Materials}

The chemical composition of the studied special aluminum alloy (containing zeolite) resembles the 6xxx series aluminum alloys, which are frequently used in industrial applications due to their low density, favorable mechanical properties, and excellent corrosion resistance. The classical definition of a zeolite is a crystalline, porous aluminosilicate. Zeolites have unusual properties under pressure. If a fluid is introduced into the tiny pores of the material, its volume increases. This extra volume can also allow slightly larger molecules or atoms, such as pollutants, to enter the expanded pores. When the pressure is released and the material contracts, the pollutants would be trapped inside. As the pressure increases, the material first compress, as one would expect. But as the pressure climbs between 0.8 and $1.5 \mathrm{GPa}$, the material expands along two of its three dimensions. When the pressure increases beyond $1.5 \mathrm{GPa}$, the material compresses once again. Analysis of the molecular structure revealed that, during the expansion, additional water molecules were added into the zeolite's pores ${ }^{17}$ A volume increase of about $7.0 \%$ occurs at pressures near $1.0 \mathrm{GPa}$ via expansions of the $a$ - and $b$-axes parameters $(4.0 \%$ and $4.2 \%$, respectively) and contraction of the $c$-axis parameter $(1.2 \%)$. Further increase of pressure above $1.2 \mathrm{GPa}$ results in the opposite behavior; i.e., the $a$ - and $b$-axes parameters contract ( $2.5 \%$ and $2.2 \%$, respectively) and the $c$-axis parameter expands $(1.2 \%)$, leading to an overall volume reduction of $3.9 \%$ compared with that of the intermediate phase. 13 Three groups of aluminum alloys of highly polished small slat each of $2 \times 2 \mathrm{~cm}^{2}$ area and $3 \mathrm{~mm}$ thickness have been investigated. The hardness of the samples has been measured by a mechanical tester.

\subsection{Method}

In the experimental setup shown in Fig. 1, the laser used is a commercial Nd:YAG laser (BRIO, Quantel, Billancourt, France) delivering laser energy of $96 \mathrm{~mJ} /$ pulse at its fundamental wavelength $\lambda=1064 \mathrm{~nm}$ with pulse duration of $5 \mathrm{~ns}$ and $20-\mathrm{Hz}$ repetition rate. The beam diameter before the focusing lens was $4 \mathrm{~mm}$, the focused beam diameter on the sample was $547.8 \mu \mathrm{m}$, and the fluence/pulse $\approx 40.75 \mathrm{~J} / \mathrm{cm}^{2}$. The laser-induced plasma was produced by focusing the laser beam by $100-\mathrm{mm}$ focal length plano-convex lens onto the sample surface, and the emission from the plasma plume was collected by a fiber optic $(600-\mu \mathrm{m}$ diameter) and fed to an echelle spectrometer (Mechelle 7500, multichannel, Stockholm, Sweden) having a spectral resolution of $0.1 \mathrm{~nm}$. The spectrometer is coupled to a computer-controlled ICCD camera (DiCAM-PRO, PCO-Computer Optics, Kelheim, Germany) with its relevant software. The lens-to-sample surface distance is controlled by a micrometer translation stage, in order to achieve precise focusing just below the target surface to avoid breakdown in the air. Each LIBS spectrum is the average of 50 spectra taken as 10 spectra at 5 different positions on each aluminum alloy sample. The obtained spectra have been displayed on a PC for further processing and spectroscopic analysis adopting LIBS++ software and relevant database. The echelle was calibrated before measurements, and we compared the spectral lines identified via LIBS++ software with NIST Spectra Database to be sure that a given emission line is from the element that we think it is from.

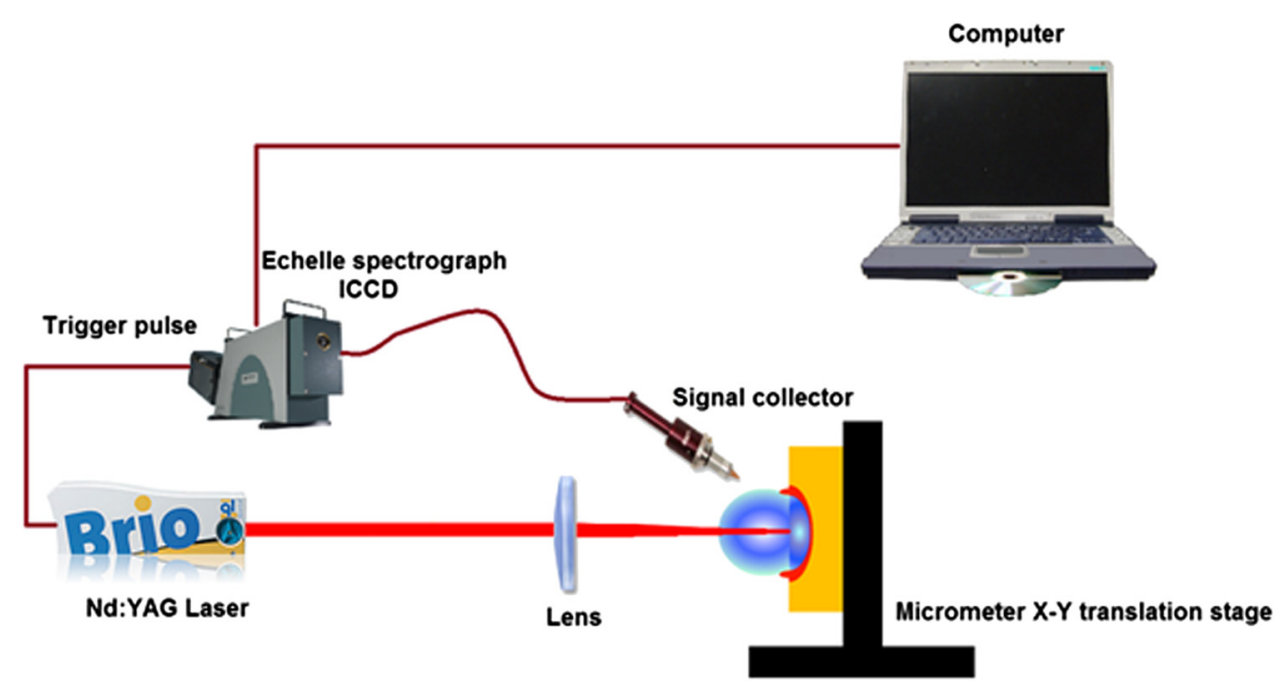

Fig. 1 Laser-induced breakdown spectroscopy (LIBS) experimental setup. 
Three identical aluminum alloy samples have been used throughout the measurements. Two of the samples are compressed to different degrees of hardness, and the third has been used as prepared. The volume and weight of the samples have been determined before and after the compression process. After the compression process, the surface hardness of the three samples was determined by Vickers hardness test and via LIBS analysis.

\section{Results and Discussions}

\subsection{LIBS and XRD Analyses}

Figure 2 shows a typical LIBS spectrum of one of the aluminum alloy samples used in the recent work. Aluminum and magnesium are the major elements, whereas $\mathrm{Ti}, \mathrm{Si}$, $\mathrm{Fe}, \mathrm{Cr}$, and $\mathrm{Co}$ represent the existing minor elements. The inset in Fig. 2(a) demonstrates the presence of zeolite, which is represented by the silicon spectral lines. The spectrum shown in Fig. 2(b) demonstrates the well-resolved magnesium lines obtained via the high-spectral resolution (better than $0.1 \mathrm{~nm}$ ) of the used echelle spectrometer-ICCD system.

To confirm the existence of aluminum silicate or "zeolite" in the samples, x-ray diffraction (XRD) spectra have been

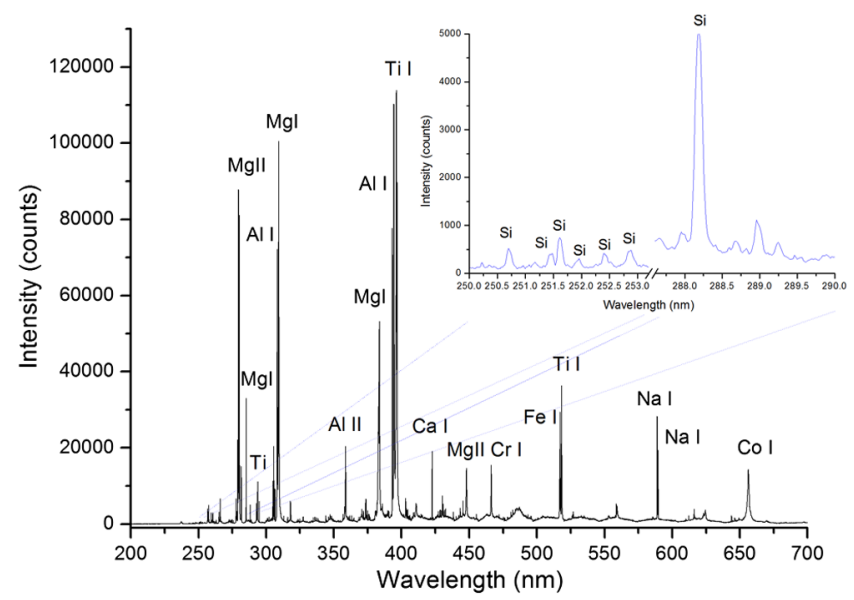

(a)

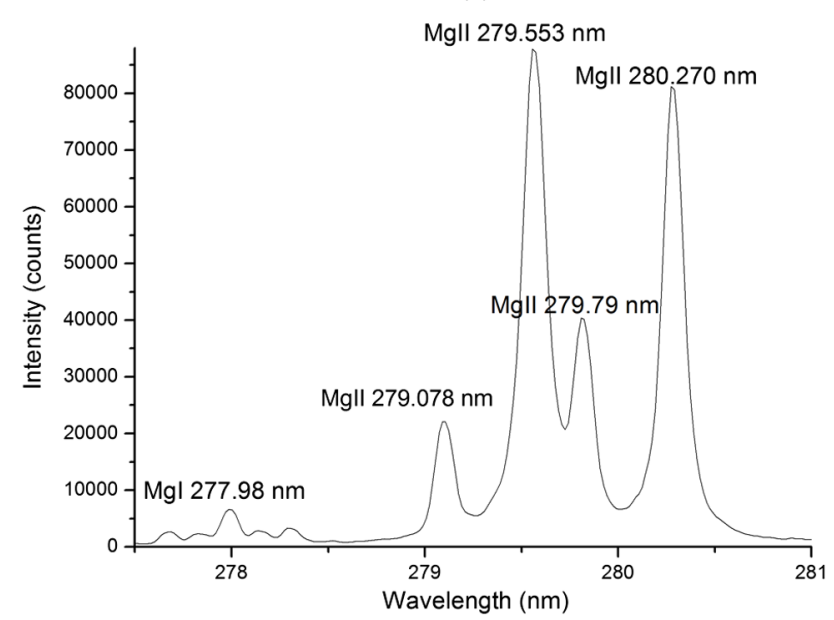

(b)

Fig. 2 (a) Typical LIBS spectrum of one of the aluminum alloy samples. The inset depicts the silicon spectral lines due to the zeolite inclusion. (b) The well-resolved magnesium spectral lines used in surface hardness determination.

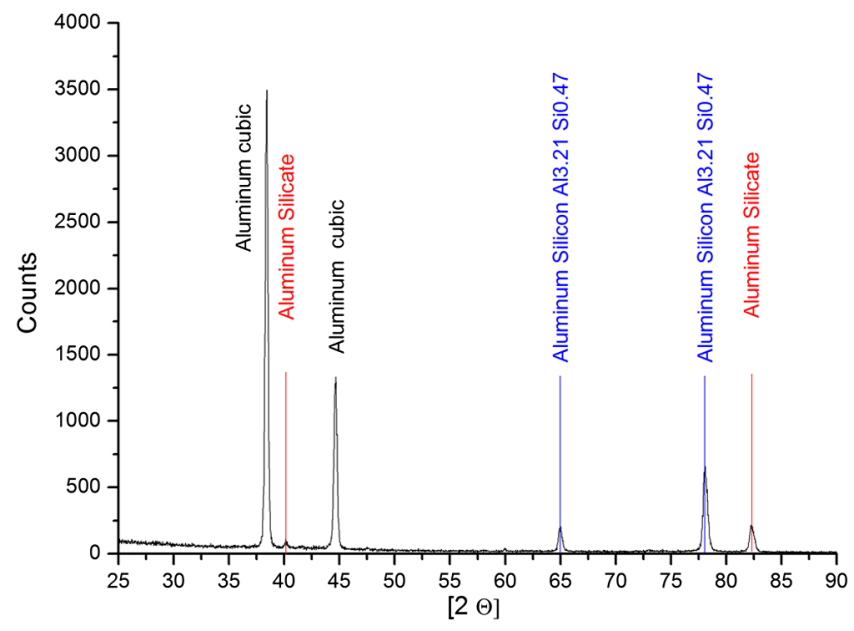

Fig. 3 X-ray diffraction (XRD) analysis for the aluminum alloy sample by X-Pert PRO, PANalytical system.

obtained for the samples under investigation. The used XRD spectrometer was an X-Pert PRO, PANalytical with fixed tube, $\mathrm{Cu}$ target, secondary graphite monochromator, flatplate horizontal sample holder, X-Celerator for faster data acquisition with X-Pert Hi-Score plus software pattern analysis, and phase identification (ICDD database). The XRD results are depicted in Fig. B, indicating the existence of the aluminum silicate $\left(\mathrm{Al}_{2} \mathrm{SiO}_{5}\right)$, beside aluminum cubic and aluminum silicon $\left(\mathrm{Al}_{3.21} \mathrm{Si}_{0.47}\right)$.

\subsection{Hardness Measurement}

In the compression test, two identical aluminum alloy samples that have been subjected to different degrees of compression suffered from a permanent change in its volume (density) after the compression procedure. The measured values of samples' hardness with conventional equipment were 87.95 HV30 for normal sample (as prepared), 104.166 HV30 for medium hardening sample, and 109.25 HV30 for maximum hardening sample.

The volume and mass of each sample are measured before and after compression procedure. The results show that the volume of the medium hardening sample has been increased by about $12 \%$, and the volume of the maximum hardening sample has been increased by about $20 \%$ after compression. This expansion in volume yields a decrease in density in the two compressed samples from $\rho$ in the normal sample to $0.893 \rho$ in the medium hardening sample and $0.833 \rho$ for the maximum hardening sample (see Fig. Đ).

Distinctively different from conventional analytical techniques, which are time consuming, labor intensive, and destructive, LIBS as an emerging analytical tool offers many superior advantages such as being fast, cheap, in situ, and a quasi-nondestructive technique. Furthermore, LIBS allows remote analysis without direct contact with the sample surface, because it requires only optical access. This would be very useful in this case, especially for analyzing difficult places or surfaces of buildings structures, where otherwise they cannot be reached by other conventional tools. $\square$ Recently, optical emission in LIBS was found to be influenced significantly by the compressive strength (hardness) of the sample, offering an opportunity for determining material hardness using LIBS technique. Accordingly, by means of 


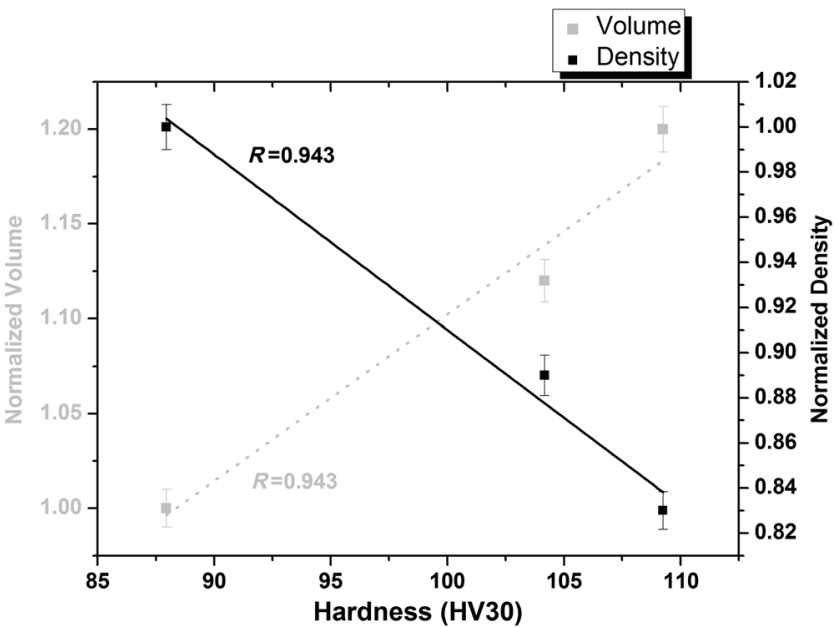

Fig. 4 Relation between sample hardness, volume, and density, demonstrating that the ratio of volume expansion with hardness increases under compression.

some specific ionic to atomic line emission ratio from the produced plasma, LIBS technique has been successfully used for measuring a specific physical property of solid, namely compressive strength of concrete.15 The developed technique was then used to measure the hardness of samples of a biological nature ${ }^{10}$ and natural minerals and stones.

To avoid a strong self-absorption effect on the lines' intensity, the partial self-absorption coefficient KR $(\lambda)$ (in $\mathrm{m}^{2} \mathrm{~s}^{-1}$ ) for a spectral line can be obtained by multiplying the self-absorption cross-section of such line $\left(S_{\mathrm{L}}\right)$ with the lower energy level population $(C)$

$\mathrm{KR}(\lambda)=S_{\mathrm{L}} \cdot C$,

where

$S_{\mathrm{L}}=0.330 \lambda_{0}^{3} \sqrt{\frac{M}{T}} \frac{g_{j}}{g_{i}} A_{j i}$,

and $C$ is given by

$C=\left(g_{j} A_{j i} / U\right) e^{-E_{i} / k T}$,

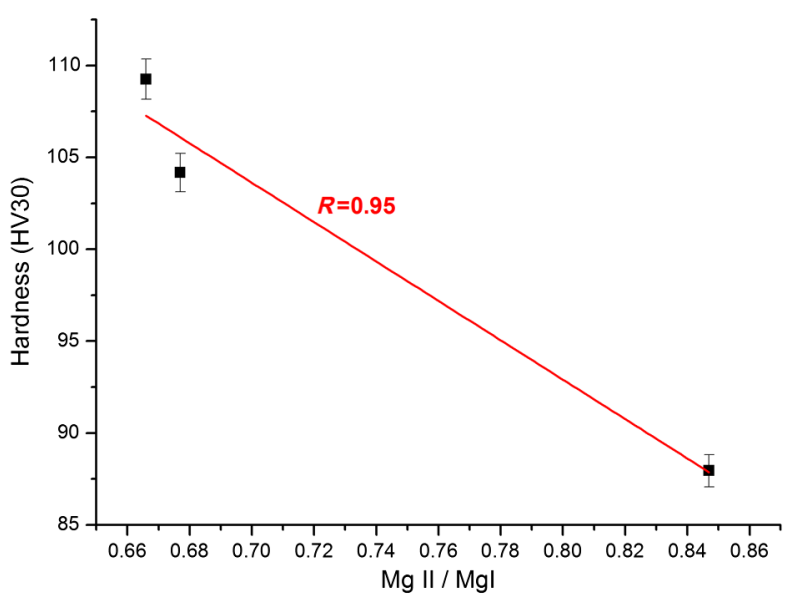

(a) where $\lambda_{0}$ is the wavelength of the spectral line (in $\mathrm{m}$ ), $T$ is the temperature (in $\mathrm{K}$ ), and $A_{j i}$ is the transition probability (in $\left.\mathrm{s}^{-1}\right) . M$ is the atomic mass of the element, $g_{i}$ and $g_{j}$ are the statistical weights of the lower and upper levels, respectively, $U$ is the partition function, $E_{i}$ is the energy of the lower energy level, and $k$ is the Boltzmann's constant. In principal, it is easy to calculate both parameters $S_{\mathrm{L}}$ and $C$, so a partial self-absorption coefficient has been calculated for each line ${ }^{\otimes}$ Since $A_{j i}$ and $g_{j}$ are in the numerator of both $S_{\mathrm{L}}$ and $C$, they have the most effect on the partial self-absorption coefficient $\mathrm{KR}(\lambda)$ final value. On the other hand, $g_{i}$ must not equal zero.

For the two magnesium spectral lines chosen in our measurements, $\mathrm{Mg}$ I $\lambda=277.983 \mathrm{~nm}\left(A_{j i}=1.36 e+08 \mathrm{~s}^{-1}\right.$, $\left.g_{j}=57833.40 \mathrm{~cm}^{-1}, \quad g_{i}=21870.464\right)$ and $\mathrm{Mg}$ II $\lambda=$ $279.078 \mathrm{~nm} \quad\left(A_{j i}=4.01 e+08 \mathrm{~s}^{-1}, g_{j}=71491.06 \mathrm{~cm}^{-1}\right.$, $\left.g_{i}=35669.31\right)$, they have relatively low-partial self-absorption coefficient KRs $(\lambda)$.

Figure 5(a) shows that the relation between the hardness and the emission intensity ratio of the aluminum alloy samples is an inverse proportionality. This result confirms that the ionic to atomic spectral lines' emission intensity ratio could be an effective measurement tool for the surface hardness. However, the relation obtained for the investigated special aluminum alloy samples demonstrates that the relation between the hardness and the emission intensity ratio of $\mathrm{Mg}$ $\mathrm{II} / \mathrm{Mg} \mathrm{I}$ is not as obtained in previous works for other materials, 1010 i.e., direct proportionality.

Figure 5(b) shows the relation between the ionic to neutral spectral lines intensity ratio and the density instead of hardness. The relation in this case is direct proportionality. So, it can be said that for these very special aluminum alloy samples, the emission intensity ratio is directly proportional to the sample density and not to its surface hardness.

The repulsive force of the laser-induced shockwaves depends clearly on the target density and laser irradiance and leads to an appreciable increase in the ionic species on the account of the neutral ones, and consequently raises the ratio of $\mathrm{Mg} \mathrm{II} / \mathrm{Mg}$ I. For low-density sample, the speed of the shockwave would be slowed down in comparison to the case of a high-density target, resulting in a reduction of the

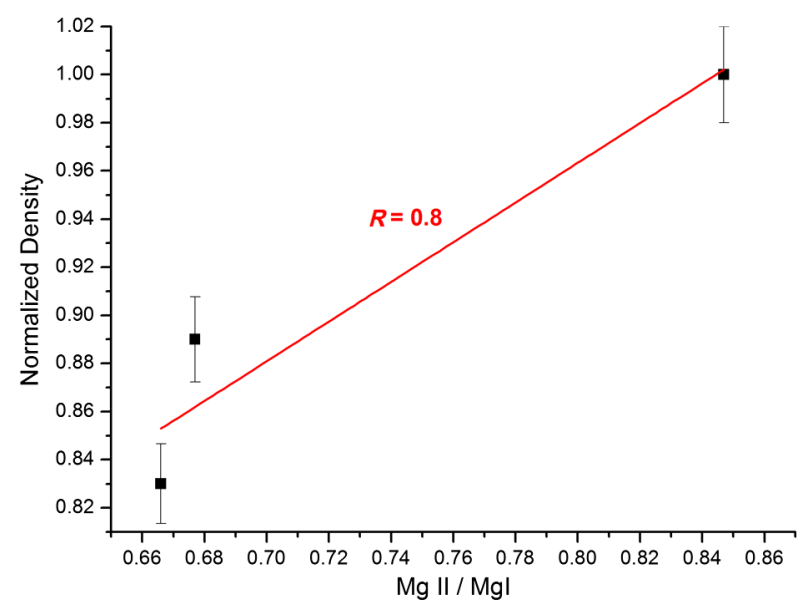

(b)

Fig. 5 (a) Correlation between hardness of aluminum alloy and the emission intensity ratio of $\mathrm{Mg} \mathrm{II}$ $279.078 \mathrm{~nm} / \mathrm{Mg}$ I $277.983 \mathrm{~nm}$. (b) Correlation between density of aluminum alloy and the emission intensity ratio of $\mathrm{Mg}$ II $279.078 \mathrm{~nm} / \mathrm{Mg}$ I $277.983 \mathrm{~nm}$. 
ionization effectiveness and then of the ionic to neutral intensity ratio. In fact, higher target density would lead to higher plasma density and consequently to higher collision frequency (which is proportional to the plasma electron density). The electrons gain energy as they oscillate in the laser field and collide with the ions, causing further ionization to the next ionic state. It is well known that this is the dominant ionization mechanism in case of nanosecond LIBS.

\section{Conclusion}

It has been demonstrated that LIBS is a powerful technique that can be used easily in the determination of the surface hardness of a metallic alloy. In the present work, special aluminum alloy samples containing zeolite have been used to determine their hardness by adopting LIBS. The idea was to relate the ionic to neutral spectral lines intensity ratio in the LIBS spectrum of the samples under study to the surface hardness of such samples. Contrary to the previously published results, the relation was inverse proportionality for such a special aluminum alloy with special metallurgical properties due to the inclusion of zeolite. However, direct proportionality between the target material density and the ionic to neutral magnesium spectral lines' intensity ratio has been obtained. This new result is relevant to the special properties acquired by the aluminum alloy due to the inclusion of zeolite.

\section{References}

1. Ron Cobden, R. T. Alcan, and R. C. Banbury, "Aluminum: physical properties, characteristics and alloys," Online lecture in "Training in Aluminium Application Technologies" By EAA (European Aluminium Association) (1994).

2. J. Fan et al., "Cyclic plasticity at pores and inclusions in cast Al-Si alloys," U. S. A. Eng. Fract. Mech. J. 70, 1281-1302 (2003).

3. "Zeolites," htm://www.highbeam.com/doc/1G1-80771077.htm (9 May 2012).

4. J. G. Martínez et al., "Meso-structured zeolite Y-high hydrothermal stability and superior FCC catalytic performance," R. Soc. Chem. J. 25, 987-994 (2012).

5. O. G. Peterson et al., "Organic dye laser threshold," J. Appl. Phys. 42(5), 1917-1928 (1971).

6. A. M. EI-nadi, M. F. Mahmoud, and H. A. Rahman, "The effect of physical parameters on the laser micro and nano melting of pure metals," New York Sci. J., 3(7), 64-69 (2010).

7. J. E. Ready, Industrial Applications of Lasers, Academic Press, New York (1978).

8. J. E. Ready, "Effects due to absorption of laser radiation," J. Appl. Phys. 36, 462-468 (1965).

9. Z. Y. Xu et al., "Pulsed laser surface hardening of ferrous alloys," in Proc. ICALEO, Section-F pp. 32-38, Laser Institute of America, Orlando, Florida (1999).

10. I. Gradshteyn and I. M. Ryzhik, Table of Integrals, Series and Products, Academic Press, New York (1980).

11. Z. Abdel Salam et al., "LIBS new application: determination of metallic alloys surface hardness," in Cairo (Egypt) AIP Conf. Proc. 1172, 49 (2009).

12. "Material that expands under pressure," http://www.bnl.gov/bnlweb/ pubaf/pr/2001/bnlpr121301.htm (9 May 2012).

13. Y. Lee et al., "Pressure-induced stabilization of ordered paranatrolite," U. S. A. Am. Mineral. J. 90, 252-257 (2005).

14. N. Idris et al., "Preliminary laser induced plasma spectroscopy (LIBS) study towards quick inspection of building quality in disaster vulnerable regions," in 5th Annual International Workshop (2010) \& Expo on Sumatra Tsunami Disaster \& Recovery, Aceh, Indonesia (2010).
15. K. Tsuyuki et al., "Measurement of concrete strength using the emission intensity ratio between $\mathrm{Ca}$ (II) $396.8 \mathrm{~nm}$ and $\mathrm{Ca}$ (I) $422.6 \mathrm{~nm}$ in a Nd:YAG laser-induced plasma," Appl. Spectrosc. J., 60(1), 60-64 (2006).

16. Z. A. Abdel-Salam et al., "Effect of experimental conditions on surface hardness measurements of calcified tissues via LIBS," Appl. Phys. B 94, 141-147 (2009).

17. N. Idris, F. Colao, and R. Fantoni, "Analytical implications of sample hardness in laser-induced breakdown spectroscopy (LIBS)," in Proc. ICONES2009, p. 321, Syiah Kuala University, Banda Aceh, Indonesia (2009).

18. Z. A. Abdel-Salam et al., "Estimation of calcified tissues hardness via calcium and magnesium ionic toatomic line intensity ratio in laser induced breakdown spectra," Spectrochim. Acta B 62, 1343-1347 (2007).

Osama Mostafa Khalil is an assistant lecturer in The High Institute of Engineering and Technology in Al Obour, Egypt. He received his BSc in electrical communication and physical engineering from Alexandria University, Egypt. He received his MSc in electronics and communications engineering from Cairo University, Egypt. Now he is a PhD student in laser physics and metallurgy, NILES Cairo University. His current research focuses on the ablation and LIBS analysis of special aluminium alloys containing zeolite under different ambient conditions.

Ilya Mingareev is a senior research scientist and a group leader at the Townes Laser Institute, the College of Optics and Photonics at the University of Central Florida. He received a PhD degree from the RWTH Aachen University in Aachen, Germany. His current research interests include laser-matter interactions, laser additive manufacturing, and various aspects of industrial laser materials processing.

Tobias Bonhoff received his BSc degree in physics from the RWTH Aachen University (Germany) in 2012. He passed a six months internship in the field of laser material processing at the Townes Laser Institute, University of Central Florida in 2012/13. Currently, he is MSc student in physics at the RWTH Aachen University. His current research focuses on the deformation of ultra-short laser pulses by optical systems.

Ashraf F. El-Sherif served as a postdoctoral research at CREOL, UCF, Orlando, Florida, from 2012 to 2013, developing a high power Tm-doped photonic crystal fiber laser. He received a $\mathrm{PhD}$ in laser photonics and modern optics from University of Manchester, United Kingdom, in 2003. He is a lecturer and faculty member at MTC, Cairo, Egypt. His current research interest is the design of new types of laser sources operating at NIR and MIR bands.

Martin C. Richardson is the director of the Townes Laser Institute and professor of optics in the College of Optics and Photonics at the University of Central Florida. He has a PhD in photon physics from London University and is credited with over 400 scientific publications in many research areas of lasers and photonics including laser-produced plasmas, laser-matter interactions, nonlinear optics, EUV/x-ray lithography, and development of new laser sources. He is a Fellow of OSA, SPIE, and an awardee of the Schardin Medal and the Harold Edgerton Award.

Mohamed Abdel Harith (BA, Physics, Cairo University 1968; PhD, Physics, TU Dresden, Germany 1976). Former dean of the National Institute of Laser Enhanced Science (NILES), Cairo University, he is currently a professor laser physics at NILES and head of the Applied Laser Spectroscopy (ALS) group. His current research interests include industrial, archaeological, and biological applications of laser induced breakdown spectroscopy (LIBS) and laser-induced fluorescence (LIF). 